\title{
Anti-Wolbachia drug discovery and development: safe macrofilaricides for onchocerciasis and lymphatic filariasis
}

\author{
MARK J. TAYLOR ${ }^{1}$, ACHIM HOERAUF ${ }^{2}$, SIMON TOWNSON $^{3}$, BARTON E. SLATKO $^{4}$ \\ and STEPHEN A. WARD ${ }^{1}$ \\ ${ }^{1}$ Liverpool School of Tropical Medicine, Pembroke Place, Liverpool, L3 5QA, UK \\ ${ }^{2}$ Institute for Medical Microbiology, Immunology and Parasitology, University Hospital Bonn, Sigmund-Freud-Strasse 25, \\ 53105 Bonn, Germany \\ ${ }^{3}$ Tropical Parasitic Diseases Unit, Northwick Park Institute for Medical Research, Watford Road, Harrow, \\ Middlesex HA1 3UF, UK \\ ${ }^{4}$ New England Biolabs, Inc., 240 County Road, Ipswich, MA 01938, USA
}

(Received 8 April 2013; revised 5 Fune 2013; accepted 5 fune 2013; first published online 18 fuly 2013)

\section{SUMMARY}

Anti-Wolbachia therapy delivers safe macrofilaricidal activity with superior therapeutic outcomes compared to all standard anti-filarial treatments, with the added benefit of substantial improvements in clinical pathology. These outcomes can be achieved, in principle, with existing registered drugs, e.g. doxycycline, that are affordable, available to endemic communities and have well known, albeit population-limiting, safety profiles. The key barriers to using doxycycline as an mass drug administration (MDA) strategy for widespread community-based control are the logistics of a relatively lengthy course of treatment (4-6 weeks) and contraindications in children under eight years and pregnancy. Therefore, the primary goal of the anti-Wolbachia (A.WOL) consortium is to find drugs and regimens that reduce the period of treatment from weeks to days (7 days or less), and to find drugs which would be safe in excluded target populations (pregnancy and children). A secondary goal is to refine regimens of existing antibiotics suitable for a more restricted use, prior to the availability of a regimen that is compatible with MDA usage. For example, for use in the event of the emergence of drug-resistance, in individuals with high loiasis co-infection and at risk of severe adverse events (SAE) to ivermectin, or in post-MDA 'endgame scenarios', where test and treat strategies become more cost effective and deliverable.

Key words: Wolbachia, onchocerciasis, lymphatic filariasis, drug discovery, macrofilaricide.

\section{INTRODUCTION}

Filariasis continues to inflict serious public health problems throughout tropical communities. The major disease-causing species include those responsible for lymphatic filariasis (LF), Wuchereria bancrofti and Brugia malayi, and onchocerciasis, Onchocerca volvulus, which together infect more than 150 million people, ranking filariasis as one of the leading causes of global morbidity (Taylor et al. 2010).

Global programmes for control and elimination have been developed to provide sustained delivery of drugs to affected communities in order to interrupt transmission of disease and ultimately eliminate this public health burden (Amazigo, 2008; Sauerbrey, 2008; WHO, 2010). Currently used drugs, diethylcarbamazine/albendazole or ivermectin/albendazole for LF and ivermectin (IVM) for onchocerciasis, principally target the microfilarial stage of the parasites and so require sustained and prolonged delivery with high treatment coverage to endemic

* Corresponding author: Head of Parasitology Department, Liverpool School of Tropical Medicine, Liverpool, L3 5QA, UK. Tel: 0044 (0)151 705 3112. Fax: 0044 (0)151 705 3371. E-mail: mark.taylor@liv.ac.uk communities in order to break the transmission cycle of the long-lived adult worms (O. volvulus, 10-14 years; $W$. bancrofti/B. malayi, 5-8 years). The impressive impact of these MDA programmes on public health is well documented (Chu et al. 2010; Coffeng et al. 2013), yet important challenges remain as these programmes translate from control to elimination goals (Bockarie and Deb, 2010; Mackenzie et al. 2012). Seventeen countries in hardto-reach areas, including post-conflict countries, have still not implemented mass drug administration (MDA) against LF 12 years after the GPELF was launched. In some of these countries, interruption of transmission will not be achieved using the current strategy alone. The growing evidence for resistance to IVM (Taylor et al. 2009; OseiAtweneboana et al. 2011) and safety constraints in areas co-endemic with Loa loa (Scientific Working Group on Serious Adverse Events in Loa Loa endemic area, 2003) has re-focused the need and urgency for new safe macrofilaricidal drugs and regimens to achieve elimination goals within existing timeframes (WHO, 2012).

Anti-Wolbachia therapy delivers safe macrofilaricidal activity with superior therapeutic outcomes compared to all standard anti-filarial treatments, with 
the added benefit of substantial improvements in clinical pathology (Taylor et al. 2010; Tamarozzi et al. 2011). These outcomes can be achieved, in principle, with existing registered drugs, e.g. doxycycline, that are affordable, available to endemic communities and have well known, albeit population-limiting, safety profiles. Anti-Wolbachia therapy delivers an early block in embryogenesis and gradual macrofilaricidal activity leading to a progressive and sustained elimination of microfilarial load, thus avoiding the risk of SAE from target species and those due to co-infections with $L$. loa (a species without Wolbachia) (Taylor et al. 2005). The use of doxycycline as a macrofilaricidal therapy has been established as proof-of-concept in an extensive series of field trials (reviewed in Hoerauf, 2008; Taylor et al. 2010), but its widespread use in community-based control is constrained by the logistics of a relatively lengthy course of treatment (4-6 weeks) and contraindications in children under eight years and pregnancy. These barriers stimulated the formation of the 'Anti-Wolbachia' (A.WOL) consortium in 2007, which was funded by the Bill \& Melinda Gates Foundation to search for new drugs active against Wolbachia that are suitable for community-directed MDA with a secondary goal to optimize regimens of existing drugs and re-purposed registered drugs for use in more restricted target populations (http://www.a-wol.net).

\section{A $\cdot$ WOL ASSAY DEVELOPMENT}

\section{Screening funnel}

As a starting point, A.WOL developed a whole organism Wolbachia cell-based assay as the primary in vitro drug-screening tool. This validated assay, which has been adapted to automated high throughput-screening and represents a rapid, sensitive and efficient assay for screening chemical libraries, utilizes a Wolbachia-containing Aedes albopictus cell line (C6/36 Wp) (Turner et al. 2006), in a 96-well format, with a quantitative PCR (qPCR) read-out to quantify the Wolbachia $16 \mathrm{~S}$ rRNA gene copy number following treatment (Johnston et al. 2010). Hits from this primary in vitro cell-based screening assay are selected based on their log drop depletion of Wolbachia, reproducibility and, if using known drugs, the target product profile (TPP) as defined by $\mathrm{A} \cdot \mathrm{WOL}$ to include oral formulation, and the safe use in children and pregnancy. These selected hits are then moved down the screening pipeline into both in vitro and in vivo nematode screening. In vitro nematode screening, using either adult male Onchocerca gutturosa ('Townson et al. 2006) or adult B. malayi, is intended to verify that hits are effective against nematode Wolbachia. These in vitro screens also identify compounds that have no direct antinematode activity yet show significant reductions in

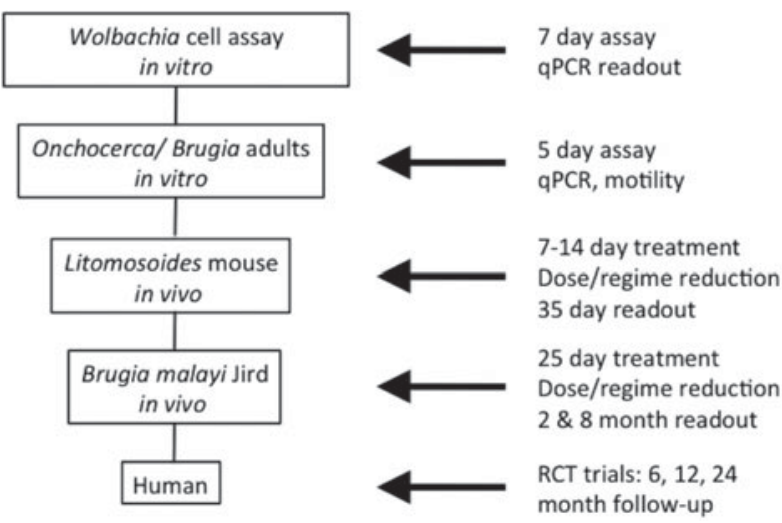

Fig. 1. Screening funnel developed for A·WOL.

Wolbachia load. For in vivo nematode screening, established animal models of filarial infection are utilized and include Litomosoides sigmodontis in mice (Hoerauf et al. 1999) and B. malayi in gerbils (Ash and Riley, 1970). For all in vivo models, the reduction of Wolbachia load following treatment is measured by qPCR. The primary in vivo screening model with $L$. sigmodontis allows for rapid screening of compounds and yields a visible and quantifiable phenotype of larvae with retarded growth. The secondary in vivo model with $B$. malayi uses a human filarial nematode and evaluates reductions in Wolbachia load predictive of macrofilaricidal activity, effects on female fertility and microfilarial production (Fig. 1).

\section{Increasing the throughput and capacity of the cell-based assay}

To date, 558000 compounds have been procured from multiple sources with $\sim 18000$ having completed screening in our standard cell-based assay with a qPCR read-out. In order to increase throughput and capacity of the A.WOL cell-based screen we have developed a 384-well format assay using a high content imaging system (Operetta) and optimization of growth dynamics in the $\mathrm{C} 6 / 36 \mathrm{~A}$. albopictus mosquito cell-line. This assay uses texture analysis of cells stained with Syto-11 as a direct measure of bacterial load and enables a shorter screening period and dramatically increases throughput and capacity. The assay has completed validation against 'hits' identified using the standard $\mathrm{qPCR}$ assay and is being used to complete diversity library screening within a 10 -fold increase in throughput (Taylor et al. unpublished results).

\section{A·WOL LIBRARY SCREENING}

One of the first activities was to develop a TPP for an $\mathrm{A} \cdot \mathrm{WOL}$ macrofilaricide. A.WOL worked with consultants from the pharma industry to compile four TPPs. Each TPP covered individual drug 
administration (IDA) or MDA for either onchocerciasis or LF. The screening campaign started with a library of registered drugs and developed to include focused libraries from pharma collaborators and large diversity-based compound libraries.

\section{Registered drug library}

Following the validation of the primary cell-based screen, the first priority was to screen approved human drug-pharmacopeia for potential repurposing for anti-Wolbachia activity. Repurposing or repositioning of drugs provides a less risky route to drug discovery given that candidates will already have well-known safety and pharmacokinetic profiles, and could provide a cost- and time-effective strategy to identify a novel A-WOL therapeutic. By screening 2664 compounds from the human drugpharmacopeia, this strategy identified 121 hits that had anti-Wolbachia activity; 69 of these were orally available from different diverse drug categories, with nine compounds being more potent than doxycycline. Several drugs have progressed further along the screening pipeline into in vitro nematode assays and in vivo screening models. The most advanced lead, minocycline has shown an increase in potency of $50 \%$ compared to doxycycline in the secondary in vivo screen and has entered efficacy trials in humans (see Drug Regimen Refinement, below; Taylor et al. unpublished results).

\section{Combination treatment}

Combinations of registered drug-screening outputs were assayed in a doxycycline enhancer assay using sub-optimal doxycycline $(50 \mathrm{~nm})$ plus 21 of the registered drug hits. These outcomes were used to design an extensive series of combinations of registered A.WOL drugs. These drugs were tested in the primary in vivo screen in triple and double combinations, with further regimen reduction experiments to determine the shortest period of treatment. The outcome of these experiments showed that in this model, double or triple combinations of registered A.WOL drugs could reduce the period treatment to 7 days or less to deliver equivalent efficacy to a standard course of doxycycline monotherapy (Specht et al. unpublished results). This outcome proved that there is no biological barrier to delivering anti-Wolbachia therapy in shortened regimens that could meet the primary goal of an $\mathrm{A} \cdot \mathrm{WOL}$ regimen compatible with MDA.

\section{Focused drug libraries}

Focused anti-infective libraries have been sourced from several pharmaceutical companies and include near-to-market lead candidate drugs or drug class derivatives which are selected from known and bio-informatically predicted essential gene targets (Holman et al. 2009, see $A \cdot W O L$ Target Discovery). Focused anti-infective library screening has, thus far, involved A.WOL in vitro screening of 3062 novel compounds from five chemical libraries. To date this has generated 184 diverse hit compounds, a number of which have progressed further into the screening funnel. Encouragingly, there is a good agreement between the reduction in Wolbachia load in the cellbased and O. gutturosa in vitro assays with no effect on worm motility. This suggests that the hits do not directly affect the nematode (and are, therefore, predicted to avoid direct parasite-mediated adverse events). Notably, the ability to identify hit compounds from these focused libraries which are effective at reducing Wolbachia load and have improved efficacy over doxycycline, is highly supportive of the long-term goal to identify A WOL new chemical entities (NCEs).

\section{Lead series originating from diversity library screens}

A screen of $>10000$ compounds from the BioFocus library revealed compounds that showed significant anti-Wolbachia activity. Retesting of these hits confirmed the identity of 50 compounds as confirmed hits (hit rate $0.5 \%$ ). Chemoinformatic analysis of these 50 hits has been used to identify the best hit series (consisting of $\sim 6$ chemotypes) with the potential to enter a medicinal chemistry 'hit to lead' and lead optimization development phase in the A.WOL II Macrofilaricide Drug Discovery programme. We have developed a rational medicinal chemistry programme around each of the six hit series. From the top six hits we have selected three templates for hit to lead optimization with three additional back-up templates. We are currently running a head to head evaluation of the three series with the intention of identifying the most promising template for final lead optimization.

Key outcomes from A.WOL library screening include the development of a portfolio of drug discovery projects with the potential to generate at least one new anti-wolbachial chemotype for eventual deployment as a macrofilaricide monotherapy (although deployment in combination would remain an option). Evidence to date suggests that there is no biological barrier to a reduced curative dosage regimen. We have already provided proofof-concept for this in experimental double/triple combination studies. Furthermore the life-style constraints of Wolbachia make acquisition of plasmidbased resistance mechanisms highly unlikely hence reducing the risk of monotherapy-driven resistance. To date several hundred 'hits' have been identified and confirmed from screening of focused 
libraries from pharma and large diversity-based libraries (Table 1).

A.WOL TARGET DISCOVERY

Targets of key enzymatic and metabolic pathways predicted from Wolbachia genomic annotation

Annotation of the $w \mathrm{Bm}$ genome suggested Wolbachia might provide haem, flavin adenine dinucleotide, riboflavin and nucleotides to the $B$. malayi host, which cannot synthesize these molecules de novo (Foster et al. 2005; Slatko et al. 2010). For example, two enzymes of the $w \mathrm{Bm}$ haem biosynthetic pathway, ALAD (aminolevulinic acid dehydratase) and $\mathrm{FeCH}$ (ferrochelatase), have been evaluated as candidate targets based on their low conservation to the corresponding human proteins, their distinct biochemical properties and sensitivities to inhibitors relative to the human enzymes. Inhibition of ALAD with succinyl acetone resulted in reduced worm motility in vitro (Wu et al. 2009) although $\mathrm{FeCH}$ is present both in Wolbachia and in the nematode genome through a lateral gene transfer event from an unrelated $\alpha$-proteobacterium (Wu et al. 2013). Also, because ALAD is not found in the genome of $B$. malayi and is significantly different from the human orthologue, it was subjected to both aptamerand chemical library-screening as part of the A.WOL programme.

Comparative genomic analyses and examination of metabolic pathway maps can indicate key differences between processes that are otherwise conserved between Wolbachia and humans leading to the identification of additional potential drug targets in Wolbachia. For example, the final step in glycolysis is catalysed by pyruvate kinase in humans but by a distinct alternative enzyme, pyruvate phosphate dikinase (PPDK), in Wolbachia (Raverdy et al. 2008). PPDK is not found in mammals. The Wolbachia PPDK enzyme has also been included in both aptamer-based and conventional library screening as part of $\mathrm{A} \cdot \mathrm{WOL}$.

$w A L A D$. The Wolbachia ALAD protein involved in the synthesis of haem was identified and validated as a target for drug screening. Direct screening of a small molecule library of 18000 compounds against the enzyme assay identified 7 compounds that inhibited wALAD compared to hALAD. Three of the compounds were based on the same benzimidazole core structure. These clustered compounds also had the highest and most specific level of inhibition of wALAD. For this reason, efforts were focused on this core structure and the best inhibitor now named wALADin 1. Characterization of the inhibitory activity has identified the mode of inhibition to be a Mixed-Model Inhibition with a calculated $\mathrm{K}_{\mathrm{I}}$ of $11 \mu \mathrm{M}$. wALADin 1 was screened in the primary cell-based assay, but unfortunately showed no activity. Activity could be demonstrated against nematodes in vitro, although only in the 0.25$0.5 \mathrm{~mm}$ range (Lentz et al. 2013). Although this work further validated haem biosynthesis as a target, in view of the more potent and tractable hits identified through library screening, further work on wALADin 1 was suspended.

PPDK. The enzymatic assay for PPDK was successfully modified for a micro-titre plate format and produced acceptable Z' values. This assay was used to screen PPDK activity against the small molecule library of 18000 compounds. Due to the large number of hits, the threshold for our cut-off for a hit was raised from $50 \%$ inhibition to $80 \%$ inhibition. 22 compounds met or surpassed this cutoff. This list was then shortened to 7 highly active compounds that appeared to be specific for PPDK (i.e. they did not inhibit wALAD). The two best compounds were found to be non-specific, therefore further development was suspended.

$L s p A$. Lipoproteins are essential structural and functional components of bacteria and those from Wolbachia are potent stimulators of the innate and adaptive inflammatory pathogenesis of filarial disease (Turner et al. 2009; Tamarozzi et al. 2011). The Wolbachia prolipoprotein signal peptidase II (LspA) was shown to be functional and the Wolbachia cellbased assay and adult B. malayi were sensitive to inhibition with a known LspA inhibitor, globomycin (Johnston et al. 2010) validating $L s p A$ as an antiWolbachia target.

\section{The 'essential gene set' of Wolbachia}

In order to target focused library screening to drugs with predicted activity against Wolbachia, a bioinformatic analysis of predicted essential genes was undertaken. An essentiality score for each predicted gene of $w \mathrm{Bm}$ was determined by two separate approaches (Holman et al. 2009). The first method compared each gene to entries in DEG (the Database of Essential Genes), a collection of $\sim 5000$ experimentally identified essential genes from 15 different bacterial species, to predict essential genes that are mostly conserved across the bacterial domain. The second approach used phyletic conservation across members of the order Rickettsiales, to which Wolbachia belongs, in order to highlight genes that are well conserved and thus likely to be essential. Conservation of genes in these rickettsial genomes that are undergoing reductive evolution underscores their importance. A ranked essentiality list was produced by each method and showed complementary and partially overlapping sets of $w \mathrm{Bm}$ genes. Many of the top-ranking genes fall into classes of 
Table 1. Summary of A.WOL screening campaign

\begin{tabular}{|c|c|c|c|c|c|c|c|c|c|}
\hline Compound class & $\begin{array}{l}\text { Screened } \\
\text { in vitro } \\
\text { cell assay }\end{array}$ & $\begin{array}{l}\text { Hits } \\
\text { in vitro }\end{array}$ & $\begin{array}{l}\text { Hits confirmed } \\
\text { using in vitro } \\
\text { worm }\end{array}$ & $\begin{array}{l}\text { Hits } \\
\text { screened } \\
\text { in } 1^{\circ} \text { in vivo }\end{array}$ & $\begin{array}{l}\text { Active }(\geqslant \text { doxy }) \\
\text { in } 1^{\circ} \text { in vivo }\end{array}$ & $\begin{array}{l}\text { Hits screened } \\
\text { in } 2^{\circ} \text { in vivo }\end{array}$ & $\begin{array}{l}\text { Active }(\geqslant \text { doxy }) \\
\text { in } 2^{\circ} \text { in vivo }\end{array}$ & $\begin{array}{l}\text { Phase II trials } \\
\text { in Ghana }\end{array}$ & $\begin{array}{l}\text { Repurposing } \\
\text { or lead } \\
\text { optimization } \\
\text { series }\end{array}$ \\
\hline \multicolumn{10}{|l|}{ Registered drug screening } \\
\hline Registered drugs & 2664 & 69 & $\checkmark$ & 20 & 4 & 2 & 1 & Minocycline & \\
\hline Registered drugs & 80 & 4 & & 3 & 0 & & & & \\
\hline Total & 2744 & 73 & & 23 & 4 & 2 & 1 & 1 & \\
\hline \multicolumn{10}{|l|}{ Focused library screening } \\
\hline Tetracyclines & 1084 & 96 & $\checkmark$ & 82 & 19 & & & & \\
\hline Boron based & 2744 & 179 & $\checkmark$ & 2 & Ongoing & 2 & Ongoing & & $\checkmark$ \\
\hline Quinolones, macrolides, NRIs & 312 & 99 & $\checkmark$ & 5 & 1 & 1 & 1 & & $\checkmark$ \\
\hline Antibacterials, kinase inhibitors & 409 & 62 & & 16 & 6 & & & & $\checkmark$ \\
\hline Active against Mtb & 1477 & 22 & & & & & & & \\
\hline Novel quinolones & 350 & 21 & Ongoing & & & & & & $\checkmark$ \\
\hline Epichem-fenarimol series & 50 & 4 & & & & & & & \\
\hline Antibacterials, anti-Mtb & 1128 & 97 & & & & & & & \\
\hline \multirow[t]{2}{*}{ Total } & 7554 & 580 & & 105 & 26 & 3 & 1 & & \\
\hline & & & & & & & & & $\checkmark$ \\
\hline Soft-focus diversity & 9946 & 112 & Ongoing & & & & & & \\
\hline Natural products & 2400 & 15 & & & & & & & \\
\hline DOS library & 924 & 2 & & & & & & & \\
\hline Diversity & 500000 & Ongoing & & & & & & & \\
\hline Diversity & 15000 & TBA & & & & & & & \\
\hline Diversity & 150000 & TBA & & & & & & & \\
\hline Total & 678270 & 129 & & nd & nd & nd & nd & & \\
\hline Grand total & 688568 & 782 & & 128 & 30 & 5 & 2 & 1 & \\
\hline
\end{tabular}


genes targeted by current antibiotics and are in functional categories predicted to be essential for bacterial growth. The high essentiality prediction of such known targets validates the computational approach. The ranked lists can be further curated to prioritize candidate drug targets by filtering for genes with no similarity to human proteins for example. The druggability of the $w \mathrm{Bm}$ proteins was addressed by comparing them to known protein targets contained within the DrugBank database, a collection of $\sim 5000$ FDA-approved small molecule drugs and compounds with details of their protein-binding partners and relevant chemical and pharmacological data. This analysis correlated well with the essential gene predictions and revealed classes of $w \mathrm{Bm}$ proteins that appear to be essential and druggable (Holman et al. 2009).

Screening of focused libraries generated with reference to the predicted essential gene list has delivered several lead compounds/drugs, which are undergoing further evaluation in $\mathrm{A} \cdot \mathrm{WOL}$ II Macrofilaricide Drug Discovery and A.WOL II Macrofilaricide Drug Development programmes.

\section{Further insight into the biological basis of Wolbachia symbiosis}

A deeper understanding of the nature of the symbiotic relationship between Wolbachia and its filarial host and the consequences of Wolbachia depletion on the biology of the nematode has advanced in recent years (Taylor et al. 2012). It now appears that the dependency of the nematodeWolbachia relationship is most critical during periods of high metabolic demands, such as larval development, growth and fertility, processes that coincide with periods of rapid Wolbachia population growth and expansion (McGarry et al. 2004; Taylor et al. 2012).

\section{Why does Wolbachia depletion induce anti-filarial activity?}

Studies on the cellular consequences of symbiont elimination have provided an important insight into the cellular mechanisms at the basis of the symbiotic relationship (Landmann et al. 2011). Soon after antibiotic elimination of the bacteria extensive apoptosis occurs in the adult germline and in the somatic cells of the embryos, microfilariae and fourth-stage larvae (L4). Apoptosis extends to uninfected cells, suggesting an indirect provision of products from the hypodermal population is required to prevent cells from undergoing cell death. This cellular mechanism does not extend to all somatic cells, including those of the hypodermal cord cells, where the bacteria reside, although the cytoskeletal arrangement is disrupted. The pattern of apoptosis activation correlates closely with the stages most vulnerable to antibiotic depletion and provides a mechanism to account for the rapid anti-filarial effects of antibiotic treatment. Additionally apoptosis signals in host nematodes could serve as useful biomarkers of anti-Wolbachia activity.

\section{Wolbachia populations are regulated by autophagy and autophagy-inducing drugs deliver bactericidal activity}

In order to understand the process by which the host nematode regulates the population growth of Wolbachia at a sufficient level to maintain the symbiosis, yet to avoid fitness costs or the pathological consequences of bacterial overgrowth, we investigated the role of autophagy, a conserved intracellular defence mechanism and regulator of cell homeostasis (Voronin et al. 2012). Activation of autophagy coincided with the onset of rapid bacterial growth and expansion, which shows that, in spite of their mutualistic association, the nematode's immune system recognises Wolbachia as a 'pathogen'. Genetic and chemical modulation of autophagy activation or suppression resulted in a corresponding decrease or increase in bacterial populations. To test whether drugs which induce the activation of autophagy could lead to a reduction in Wolbachia populations in vivo, we treated jirds infected with $B$. malay $i$ with rapamycin and spermidine. Treatment with rapamycin or spermidine reduced Wolbachia loads by $\sim 70 \%$ for both drugs compared to the control (Voronin et al. 2012). These results provide proof-of-concept that drug-induced activation of autophagy is effective at reducing Wolbachia populations in vivo to the same extent as antibiotic therapy and identifies a novel bactericidal mode-of-action which can be exploited in the discovery and development of new antiWolbachia treatments.

\section{A. WOL REGIMEN REFINEMENT}

In order to address A.WOL's second goal to optimize regimens of known anti-wolbachial drugs (doxycycline and rifampicin), we carried out a series of phase II field trials with the aim of testing the efficacy of reduced dosage (200 to $100 \mathrm{mg}$ ) and to test whether combinations of anti-wolbachial drugs can reduce the treatment period. Two additional studies were initiated to pilot the lead candidate from our registered library screen (minocycline) and to evaluate the efficacy of community-directed doxycycline treatment four years after delivery. (1) A.WOL LF I: RCT phase II trial, doxycycline vs doxycycline/rifampicin and doxycycline dose reduction (200 to $100 \mathrm{mg}$ ), Ghana. (2) A.WOL oncho I: RCT phase II trial, doxycycline $v$ s doxycycline/rifampicin and doxycycline dose reduction 
(200 to $100 \mathrm{mg}$ ), Ghana. (3) A.WOL oncho II: Open label pilot trial, doxycycline $v s$ minocycline \pm albendazole, Ghana. (4) A.WOL oncho III: Evaluation of the effectiveness of communitydirected delivery of doxycycline four years after delivery (Cameroon).

All follow-up sampling of phase II and pilot trials is now complete, with ongoing laboratory analysis of primary and secondary endpoints underway, which is expected to be completed by the end of 2013 .

In 2007 and 2008, a feasibility trial of communitydirected treatment with doxycycline was carried out in two health districts in Cameroon, co-endemic for O. volvulus and L. loa (Wanji et al. 2009). With 17519 eligible subjects, the therapeutic coverage was $73 \cdot 8 \%$ with $97.5 \%$ compliance, encouraging the feasibility of using doxycycline community-directed delivery in restricted populations of this size. The effectiveness of this community-directed delivery of doxycycline was further evaluated four years after delivery (Tamarozzi et al. 2012). Statistically significant lower microfilarial prevalence $(17 \cdot 0 \%$ [doxycycline plus ivermectin group], $27 \cdot 0 \%$ [ivermectin only group], $P=0.014)$ and load $(P=0.012)$ were found in people that had received doxycycline followed by ivermectin compared to those who received ivermectin only. This study demonstrates the long-term effectiveness of doxycycline treatment delivered with a community-directed strategy even when evaluated four years after delivery in an area of ongoing transmission. This finding shows that a multi-week course of treatment is not a barrier to community-delivery of MDA in restricted populations of this size and supports its implementation to complement existing control strategies for onchocerciasis, where needed ('Tamarozzi et al. 2012).

\section{A. WOL MATHEMATICAL MODELLING}

An extensive series of trials has shown that doxycycline treatment eliminates Wolbachia causing long-term sterilization of adult female filariae and ultimately exerting a macrofilaricidal effect against onchocerciasis and LF. Such trials have been conducted in endemic settings where continual reinfection by drug-naïve worms compromises the evaluation of macrofilaricidal efficacy (Specht et al. 2009). This makes it difficult to estimate therapeutic efficacy and compare data from different doxycycline regimens collected at different times post-treatment. A mathematical model was developed which couples the doxycycline-induced depletion of Wolbachia from adult $O$. volvulus to the ensuing macrofilaricidal activity (Walker et al. unpublished results). The model was fitted to data from clinical trials measuring the Wolbachia status and viability of individual female adult worms exposed to a 4-, 5- or 6-week daily dose of 100 or $200 \mathrm{mg}$ oral doxycycline. Doxycycline induces rapid depletion of Wolbachia, yet these effects are most apparent 9.5 months after the start of treatment. The estimated therapeutic efficacy of doxycycline in eliminating Wolbachia from female $O$. volvulus increases statistically significantly from 92 to $95 \%$ from 4 to 5 weeks of treatment and non-significantly from 95 to $97 \%$ from 5 to 6 weeks of treatment, irrespective of dose. This model validates the marked macrofilaricidal activity of doxycycline therapy and provides robust statistical support for equivalent efficacy with reduced timeframes and dosage and can be adapted to the analysis of other A.WOL therapies for the treatment of both onchocerciasis and LF.

ANTI - WOLBACHIA TREATMENT IMPROVES CLINICAL DISEASE

Previously, a course of doxycycline was shown not only to possess macrofilaricidal activity, but also lead to significant clinical improvements in the severity of lymphoedema (Debrah et al. 2006). In a second trial this outcome was compared with a course of amoxicillin and in patients without active LF infection (Mand et al. 2012). Doxycycline-treated patients with lymphoedema (LE) stage 2-3 showed significant reductions in LE severity after 12 and 24 months, regardless of circulating filarial antigen status. Improvement was observed in $43.9 \%$ of doxycycline-treated patients, compared with only $3 \cdot 2$ and $5 \cdot 6 \%$ in the amoxicillin and placebo arms, respectively. Both doxycycline and amoxicillin reduced acute dermatolymphangioadenitis attacks. This unexpected outcome showed that improvements in lymphoedema were also found in patients without active infection, which expands the use of this approach as a new and improved tool for morbidity management as part of GPELF (Mand et al. 2012).

\section{CONCLUSIONS}

A.WOL has developed a series of validated and robust assays to evaluate drugs and compounds with anti-Wolbachia activity which have been used to screen a range of registered, focused and diversity drug libraries to deliver several hundred 'hits', which are progressing through the screening funnel with the potential to generate at least one new anti-wolbachial chemotype for eventual deployment as a macrofilaricide. The outcomes of the initial A.WOL programme are progressing through $\mathrm{A} \cdot \mathrm{WOL}$ II Macrofilaricide Drug Discovery and A.WOL II Macrofilaricide Drug Development programmes.

Regimens of known A.WOL drugs have been optimised for dosage and time-frame to deliver a curative course of treatment equivalent to regimens for prophylaxis for traveller's malaria and acne which can be considered, in restricted populations, to complement existing MDA strategies in 'hot spot' 
foci or residual populations in MDA end-game scenarios, where test and treat strategies become more cost-effective and deliverable than MDA. An example is the decision by Onchocerciasis Elimination Programme for the Americas (OEPA) to use doxycycline in attempts to foreshorten the time to elimination in a focus in Venezuela to achieve the Regional Elimination goal. The WHO Road Map (WHO, 2012) at the time of the commitment to the London Declaration on NTDs has ambitious targets based on existing available preventive chemotherapy tools. However, it is recognized that if there is to be a more rapid drive towards elimination and strategies to tackle the barriers of reduced efficacy of existing drugs and L. Loa, A.WOL outcomes should be considered for deployment at the earliest opportunity.

\section{ACKNOWLEDGEMENTS}

We thank all of the A.WOL consortium partners and their laboratory and field trial teams.

\section{F I NANCIAL SUPPORT}

The A.WOL consortium is supported by a grant from the Bill \& Melinda Gates Foundation award to the Liverpool School of Tropical Medicine.

\section{REFERENCES}

Amazigo, U. (2008). The African Programme for Onchocerciasis Control (APOC). Annals of Tropical Medicine and Parasitology 102(Suppl 1), 19-22. doi: 10.1179/136485908X337436.

Ash, L. R. and Riley, J. M. (1970). Development of subperiodic Brugia malayi in the jird, Meriones unguiculatus, with notes on infections in other rodents. Fournal of Parasitology 56, 969-973.

Bockarie, M. J. and Deb, R. (2010). Elimination of lymphatic filariasis: do we have the drugs to complete the job? Current Opinion in Infectious Diseases 23, 617-620. doi: 10.1097/QCO.0b013e32833fdee5.

Chu, B. K., Hooper, P.J., Bradley, M.H., McFarland, D. A. and Ottesen, E. A. (2010). The economic benefits resulting from the first 8 years of the Global Programme to Eliminate Lymphatic Filariasis (2000-2007). PLoS Neglected Tropical Diseases 4, e708. doi: 10.1371/journal. pntd.0000708.

Coffeng, L. E., Stolk, W. A., Zouré, H. G., Veerman, J. L., Agblewonu, K. B., Murdoch, M.E., Noma, M., Fobi, G., Richardus, J.H., Bundy, D. A., Habbema, D., de Vlas, S. J. and Amazigo, U. V. (2013). African Programme For Onchocerciasis Control 1995-2015: model-estimated health impact and cost. PLoS Neglected Tropical Diseases 7, e2032. doi: 10.1371/journal.pntd.0002032.

Debrah, A. Y., Mand, S., Specht, S., Marfo-Debrekyei, Y., Batsa, L., Pfarr, K., Larbi, J., Lawson, B., Taylor, M., Adjei, O. and Hoerauf, A. (2006). Doxycycline reduces plasma VEGF-C/sVEGFR-3 and improves pathology in lymphatic filariasis. PLoS Pathogens 2, e92.

Foster, J., Ganatra, M., Kamal, I., Ware, J., Makarova, K., Ivanova, N., Bhattacharyya, A., Kapatral, V., Kumar, S., Posfai, J., Vincze, T., Ingram, J., Moran, L., Lapidus, A., Omelchenko, M., Kyrpides, N., Ghedin, E., Wang, S., Goltsman, E., Joukov, V., Ostrovskaya, O., Tsukerman, K., Mazur, M., Comb, D., Koonin, E. and Slatko, B. (2005). The Wolbachia genome of Brugia malayi: endosymbiont evolution within a human pathogenic nematode. PLoS Biology 3, e121.

Hoerauf, A. (2008). Filariasis: new drugs and new opportunities for lymphatic filariasis and onchocerciasis. Current Opinion in Infectious Disease 21, 673-681. doi: 10.1097/QCO.0b013e328315cde7.

Hoerauf, A., Nissen-Pähle, K., Schmetz, C., Henkle-Dührsen, K., Blaxter, M.L., Büttner, D. W., Gallin, M.Y., Al-Qaoud, K. M.,
Lucius, R. and Fleischer, B. (1999). Tetracycline therapy targets intracellular bacteria in the filarial nematode Litomosoides sigmodontis and results in filarial infertility. Fournal of Clinical lavestigation 103, 11-18.

Holman, A. G., Davis, P. J., Foster, J. M., Carlow, C. K. and Kumar, S. (2009). Computational prediction of essential genes in an unculturable endosymbiotic bacterium, Wolbachia of Brugia malayi. BMC Microbiology 9, 243.

Johnston, K. L., Wu, B., Guimaraes, A., Ford, L., Slatko, B. E. and Taylor, M. J. (2010). Lipoprotein biosynthesis as a target for antiWolbachia treatment of filarial nematodes. Parasites and Vectors 3, 99. Landmann, F., Voronin, D., Sullivan, W. and Taylor, M. J. (2011) Anti-filarial activity of antibiotic therapy is due to extensive apoptosis after Wolbachia depletion from filarial nematodes. PLoS Pathogens 7, e1002351. Lentz, C.S., Halls, V., Hannam, J.S., Niebel, B., Strübing, U., Mayer, G., Hoerauf, A., Famulok, M. and Pfarr, K. M. (2013). A selective inhibitor of heme biosynthesis in endosymbiotic bacteria elicits antifilarial activity in vitro. Chemical Biology 20, 177-187. doi: 10.1016/j. chembiol.2012.11.009.

Mackenzie, C.D., Homeida, M. M., Hopkins, A.D. and Lawrence, J. C. (2012). Elimination of onchocerciasis from Africa: possible? Trends in Parasitology 28, 16-22. doi: 10.1016/j.pt.2011.10.003.

Mand, S., Debrah, A.Y., Klarmann, U., Batsa, L., MarfoDebrekyei, Y., Kwarteng, A., Specht, S., Belda-Domene, A., Fimmers, R., Taylor, M., Adjei, O. and Hoerauf, A. (2012). Doxycycline improves filarial lymphedema independent of active filarial infection: a randomized controlled trial. Clinical Infectious Diseases 55, 621-630. doi: 10.1093/cid/cis486.

McGarry, H. F., Egerton, G. and Taylor, M. J. (2004). Population dynamics of Wolbachia bacterial endosymbionts in Brugia malayi. Molecular and Biochemical Parasitology 135, 57-67.

Osei-Atweneboana, M. Y., Awadzi, K., Attah, S. K., Boakye, D. A., Gyapong, J. O. and Prichard, R. K. (2011). Phenotypic evidence of emerging ivermectin resistance in Onchocerca volvulus. PLoS Neglected Tropical Diseases 5, e998.

Raverdy, S., Foster, J. M., Roopenian, E. and Carlow, C. K. (2008). The Wolbachia endosymbiont of Brugia malayi has an active pyruvate phosphate dikinase. Molecular and Biochemical Parasitology 160, 163-166. doi: 10.1016/j.molbiopara.2008.04.014.

Sauerbrey, M. (2008). The Onchocerciasis Elimination Program for the Americas (OEPA). Annals of Tropical Medicine and Parasitology 102(Suppl 1), 25-29. doi: 10.1179/136485908X337454

Scientific Working Group on Serious Adverse Events in Loa Loa endemic areas (2003). Report of a Scientific Working Group on Serious Adverse Events following Mectizan(R) treatment of onchocerciasis in Loa loa endemic areas. Filaria fournal 2(Suppl 1), S2.

Slatko, B.E., Taylor, M. J. and Foster, J. M. (2010). The Wolbachia endosymbiont as an anti-filarial nematode target. Symbiosis 51, 55-65.

Specht, S., Hoerauf, A., Adjei, O., Debrah, A. and Büttner, D. W. (2009). Newly acquired Onchocerca volvulus filariae after doxycycline treatment. Parasitology Research 106, 23-31. doi: 10.1007/s00436-0091624-5.

Tamarozzi, F., Halliday, A., Gentil, K., Hoerauf, A., Pearlman, E. and Taylor, M. J. (2011). Onchocerciasis: the role of Wolbachia bacterial endosymbionts in parasite biology, disease pathogenesis, and treatment. Clinical Microbiology Reviews 24, 459-468. doi: 10.1128/CMR.00057-10. Tamarozzi, F., Tendongfor, N., Enyong, P. A., Esum, M., Faragher, B., Wanji, S. and Taylor, M. J. (2012). Long term impact of large scale community-directed delivery of doxycycline for the treatment of onchocerciasis. Parasite and Vectors 5, 53.

Taylor, M. J., Bandi, C. and Hoerauf, A. (2005). Wolbachia bacterial endosymbionts of filarial nematodes. Advances in Parasitology 60, 245-284. Taylor, M. J., Awadzi, K., Basáñez, M. G., Biritwum, N., Boakye, D., Boatin, B., Bockarie, M., Churcher, T. S., Debrah, A., Edwards, G., Hoerauf, A., Mand, S., Matthews, G., Osei-Atweneboana, M., Prichard, R. K., Wanji, S. and Adjei, O. (2009). Onchocerciasis control: vision for the future from a Ghanian perspective. Parasites and Vectors 2, 7 .

Taylor, M. J., Hoerauf, A. and Bockarie, M. (2010). Lymphatic filariasis and onchocerciasis. Lancet 376, 1175-1185.

Taylor, M. J., Voronin, D., Johnston, K. L. and Ford, L. (2012). Wolbachia filarial interactions. Cellular Microbiology 15, 520-626. doi: 10.1111/cmi.12084.

Townson, S., Tagboto, S., McGarry, H.F., Egerton, G. L. and Taylor, M. J. (2006). Onchocerca parasites and Wolbachia endosymbionts: evaluation of a spectrum of antibiotic types for activity against Onchocerca gutturosa in vitro. Filaria Fournal 5, 3 .

Turner, J. D., Langley, R. S., Johnston, K. L., Egerton, G. L., Wanji, S. and Taylor, M. J. (2006). Wolbachia endosymbiotic bacteria of Brugia 
malayi mediate macrophage tolerance to TLR and CD40 specific stimuli in a TLR2/MyD88 dependent manner. Fournal of Immunology 7, 1240-1249. Turner, J. D., Langley, R.S., Johnston, K. L., Gentil, K., Ford, L., Wu, B., Graham, M., Sharpley, F., Slatko, B., Pearlman, E. and Taylor, M. J. (2009). Wolbachia lipoprotein stimulates innate and adaptive immunity through toll-like receptors 2 and 6 (TLR2/6) to induce disease manifestations of filariasis. Fournal of Biological Chemistry 284, 22364-22378.

Voronin, D., Cook, D. A., Steven, A. and Taylor, M. J. (2012). Autophagy regulates Wolbachia populations across diverse symbiotic associations. Proceedings of the National Academy of Sciences, USA 109, E1638-E1646.

Wanji, S., Tendongfor, N., Nji, T., Esum, M., Che, J. N., Nkwescheu, A., Alassa, F., Kamnang, G., Enyong, P. A., Taylor, M. J., Hoerauf, A. and Taylor, D. W. (2009). Communitydirected delivery of doxycycline for the treatment of onchocerciasis in areas of co-endemicity with loiasis in Cameroon. Parasite and Vectors 2, 39.
Wu, B., Novelli, J., Foster, J., Vaisvila, R., Conway, L., Ingram, J., Ganatra, M., Rao, A. U., Hamza, I. and Slatko, B. (2009). The heme biosynthetic pathway of the obligate Wolbachia endosymbiont of Brugia malayi as a potential anti-filarial drug target. PLoS Neglected Tropical Diseases 3, e475. doi: 10.1371/journal.pntd.0000475.

Wu, B., Novelli, J., Jiang, D., Harry, A., Dailey, H. A., Landmann, F., Ford, L., Taylor, M. J., Carlow, C. K. S., Foster, J. M. and Slatko, B. E. (2013). Interdomain lateral gene transfer of an essential ferrochelatase gene in human parasitic nematodes. Proceedings of the National Academy of Sciences, USA 110, $7748-7753$.

World Health Organisation (2010). GPELF Progress Report 2000-2009 and Strategic Plan 2010-2020. WHO/HTM/NTD/PCT/ 2010.6 .

World Health Organisation (2012). Accelerating work to overcome the global impact of neglected tropical diseases - A roadmap for implementation WHO/HTM/NTD/2012.1. 